\title{
Beyond Terabit/s WDM Optical Wireless Transmission using Wavelength-transparent Beam Tracking and Steering
}

\author{
Yang Hong ${ }^{1}$, Feng Feng ${ }^{2}$, Kyle R. H. Bottrill ${ }^{1}$, Natsupa Taengnoi ${ }^{1}$, Ravinder Singh ${ }^{2}$, Grahame Faulkner ${ }^{2}$, \\ Dominic C. O'Brien ${ }^{2}$ and Periklis Petropoulos ${ }^{1}$ \\ 1 Optoelectronics Research Centre, University of Southampton, Southampton SO17 1BJ, United Kingdom \\ 2 Department of Engineering Science, University of Oxford, Oxford OX1 3PJ, United Kingdom \\ Authore-mail address: y.hong@soton.ac.uk
}

\begin{abstract}
We report up to $1.165-\mathrm{Tb} / \mathrm{s}$ optical wireless WDM transmission using a wavelengthtransparent beam tracking and steering system. Over a $3.5-\mathrm{m}$ perpendicular distance, beyond 1$\mathrm{Tb} / \mathrm{s}$ capacity was achieved across a lateral coverage up to $1.8 \mathrm{~m}$. (C) 2020 The Author(s)
\end{abstract}

\section{Introduction}

Optical wireless communications (OWC) has attracted significant interest in recent years, largely owing to its benefits relating to the use of an unregulated bandwidth, immunity to electromagnetic interference and the potential for achieving vast transmission rates [1-5]. In broad terms, OWC can be divided into two categories: visible light communication (VLC) and infrared OWC. By directly modulating visible light-emitting diodes (LEDs), VLC offers simultaneous illumination and data transmission. Although by their nature, LEDs can offer a broad coverage, their transmission capability is relatively limited [2]. Increasing data rate usually requires lasers rather than LEDs, as these can be modulated at higher frequencies, and results in narrow field-of view optical links. The restricted field of view is required to minimize link loss, as at high data rates receiver sensitivity reduces, and modulated source power is also more limited [3]. Therefore, beam tracking and steering is a critical issue in such systems [1]. The various solutions to address this issue have included the use of optical phased arrays [4] or an arrayed waveguide grating router (AWGR) [1]. While employing an AWGR avoids the requirement for beam tracking and allows a high overall throughput to the optical wireless channel, it does not easily lend itself to wavelength-division multiplexing (WDM) operation and the delivery of ultrahigh capacity transmission to a single receiver, since different wavelengths are steered to different locations [1].

Using a system that employs a camera-based tracking and steering, high-speed WDM transmission using the 4ary pulse amplitude modulation was recently demonstrated in [5]. In this paper, we extend the transmission capacity of this system while remaining within the eye-safety power limits, and report the delivery of up to $1.165-\mathrm{Tb} / \mathrm{s}$ adaptively-loaded discrete multitone (DMT) modulation WDM signals to a single optical wireless receiver over a distance of $3.5 \mathrm{~m}$. Thanks to the wavelength-transparent property of the mirror-based steering system, similar transmission performance was achieved for all ten WDM channels. Furthermore, we confirmed that beyond $1-\mathrm{Tb} / \mathrm{s}$ transmission at a bit-error rate (BER) lower than $3.8 \times 10^{-3}$ was achieved across a lateral distance of up to $1.8 \mathrm{~m}$.

\section{Experimental Setup}

Fig. 1(a) shows the block diagram of the developed beam tracking and steering system. Beam steering is based on a set of mirrors (M1 and M2), which include rings of LEDs to serve as beacons (BCN1 and BCN2) for tracking. Each of the transmitter (Tx) and receiver (Rx) includes two cameras, one of which has a wide field-of-view (FOV), i.e., CAM1 and CAM3, while the second has a narrow FOV, i.e., CAM2 and CAM4. The wide FOV cameras first find the rough positions of the Tx and Rx, followed by fine tracking achieved by the two narrow FOV cameras, in front of which two bandpass filters (BFs) are adopted to filter out ambient light, respectively. The gathered location information is then used to control the steering mirror to achieve beam steering. The LEDs in BCN1 and BCN2 emit at different wavelengths ( $800 \mathrm{~nm}$ and $890 \mathrm{~nm}$, respectively), to avoid interference between the Tx and Rx during the tracking process. At the Tx, the data signal is launched through an optical fiber collimator (Col1), followed by a dichroic filter (DF). A similar configuration is used at the Rx, and the data signal alone is collected through collimator Col2. Since beam steering is achieved by mirrors, the system is wavelength-transparent, which facilitates the potential of WDM transmission for ultrahigh-speed OWC.

To demonstrate the tracking and steering capability of the system, we implemented an OWC system (Fig. 1(b\& c). We fixed the location of the Tx whilst laterally moving the Rx. The vertical distance between Tx and Rx was 3.5 $\mathrm{m}$. Due to the limited space available for the demonstration, only right-side lateral movement (up to $0.9 \mathrm{~m}$ ) was tested, (see Fig.1(b)). Considering the symmetrical properties of the optics and tracking cameras, a full tracking 
coverage of $1.8 \mathrm{~m}$ can be assumed. Ten 100-GHz-spaced WDM channels ranging from $1546.12 \mathrm{~nm}$ to $1553.33 \mathrm{~nm}$ were used as optical carriers, and the Mach-Zehnder modulator (MZM) was modulated by adaptively-loaded DMT signals that were generated by a 70-GSa/s arbitrary waveform generator (AWG). The IFFT/FFT size of the DMT signals was 512, out of which 240 carriers were used for data modulation, hence the signal bandwidth was 32.8125 GHz. The modulated optical signals in odd and even WDM channels were demultiplexed in a Waveshaper and decorrelated by passing through different lengths of fiber. Subsequently, they were amplified by a booster erbiumdoped fiber amplifier (EDFA) and the incident optical power to the Tx of the beam tracking and steering system was attenuated to $9 \mathrm{dBm}$ for eye safety. At the receiver side, a pre-amp EDFA was adopted at the output of the Rx. An optical bandpass filter (OBPF) was then used to select each of the WDM channels, followed by an optical attenuator to vary the received optical power (ROP) at the photodetector (PD). The detected electrical signals at the PD were then captured by an $80-\mathrm{GSa} / \mathrm{s}$ digital storage oscilloscope (DSO) for offline digital signal processing.

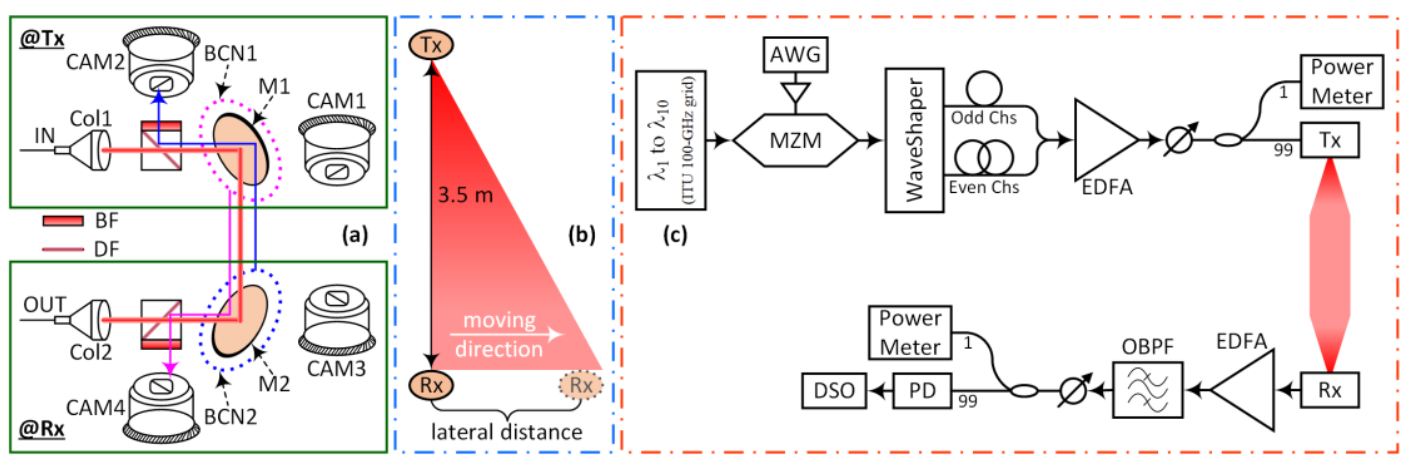

Fig. 1. (a) Diagram of the camera-and-mirror based beam tracking and steering system, (b) illustration of the relative placement of the Tx and Rx, and (c) experimental setup of the WDM OWC system.

\section{Experimental Results}

We firstly evaluated the OWC system when the Tx and Rx were vertically aligned, i.e., the lateral distance shown in Fig. 1(b) was $0 \mathrm{~m}$. The link alignment was optimized to ensure a minimum loss $(\sim 10.5 \mathrm{~dB})$ of the optical wireless link, and thus an optimal transmission performance. The corresponding maximum capacities of all ten WDM channels versus ROP for a BER below $3.8 \times 10^{-3}$ (the 7\% FEC limit) are shown in Fig. 2(a). Note that to obtain these capacity values, pilot transmission was conducted for all channels under each ROP to estimate the respective signalto-noise ratio (SNR) condition, which was then used for adaptive bit-and-power loading. Thanks to the wavelengthtransparent property of the tracking and steering system, comparable performance was achieved for all WDM channels across all ROP values we tested, see Fig. 2(a). Furthermore, it is seen that when ROP is >-11 dBm, beyond $100 \mathrm{~Gb} / \mathrm{s}$ transmission can be achieved for all channels. Fig. 2(b) plots the maximum capacity of all ten WDM channels for a ROP of $-7 \mathrm{dBm}$. All channels achieved a capacity of $\sim 116 \mathrm{~Gb} / \mathrm{s}$ whilst maintaining a BER below the $7 \%$ FEC limit. At this power level, the aggregate maximum capacity of the WDM OWC system was $\sim 1.165 \mathrm{~Tb} / \mathrm{s}$.
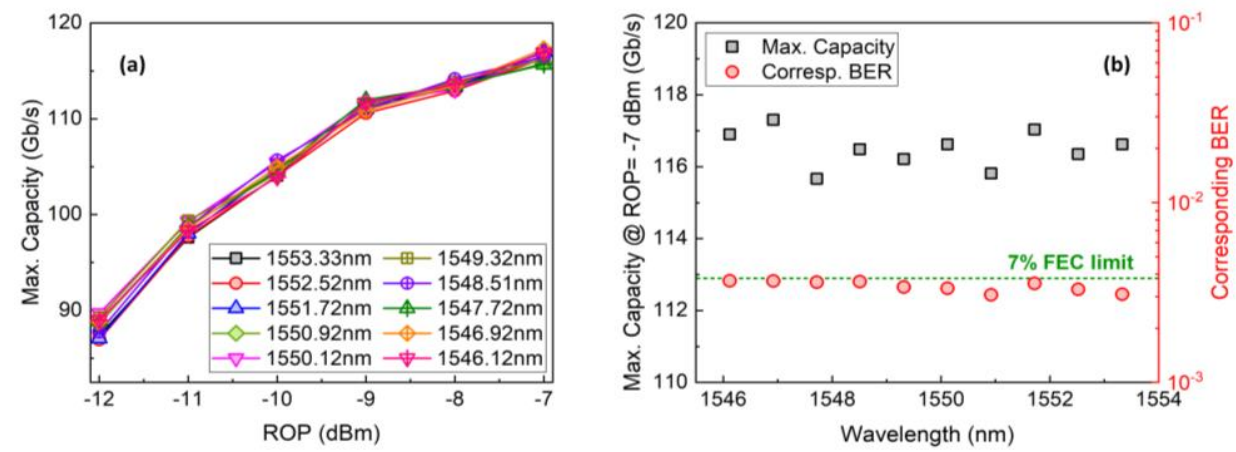

Fig. 2. (a) Maximum capacity versus ROP of the OWC system at a lateral distance of $0 \mathrm{~m}$, and (b) Capacity of all ten channels at a ROP of $-7 \mathrm{dBm}$ and their corresponding BERs.

We further validated the tracking capability of the tracking and steering system by laterally moving the Rx along a straight line. As shown in Fig. 3(a), by only using the camera-based tracking, the loss of the optical wireless link increased from $10.5 \mathrm{~dB}$ to $17 \mathrm{~dB}$ when the lateral distance increased from $0 \mathrm{~m}$ to $0.9 \mathrm{~m}$. For reference, the losses of 
the link obtained by manual optimization are also given in Fig. 3(a), which show relatively uniform performance across the lateral coverage. Here, manual optimization refers to two-dimensional scanning of all possible angles of the steering mirror to identify the optimal link alignment which achieves the minimum loss.
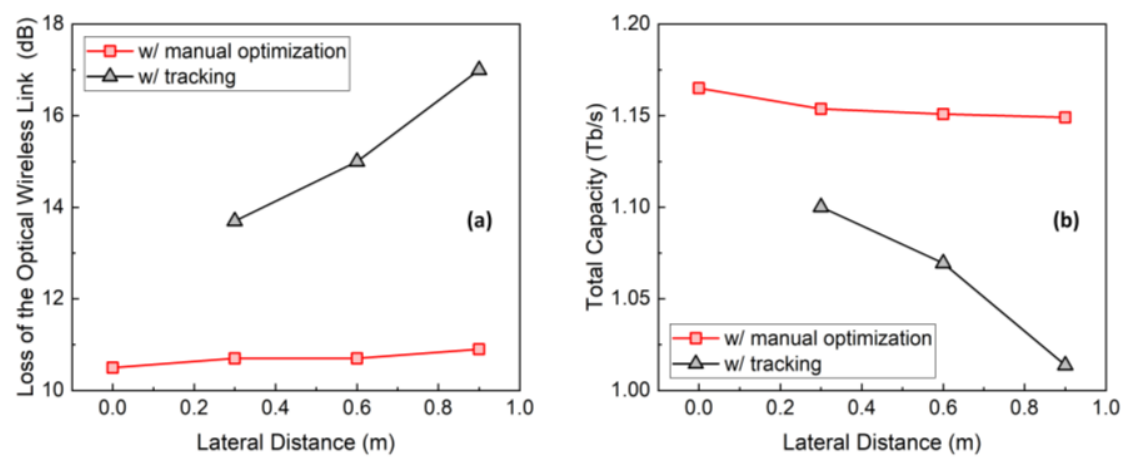

Fig. 3. (a) Loss of the optical wireless link versus lateral distance, and (b) total capacity versus later distance.

The corresponding impact of the lateral movement on the total capacity of the OWC system is shown in Fig. 3(b). It is seen that the camera-based tracking and steering system achieves beyond 1-Tb/s transmission capacity for up to $0.9-\mathrm{m}$ (single-side) lateral movement, i.e. a full lateral coverage of $1.8 \mathrm{~m}$. By adopting manual optimization, around $1.15-\mathrm{Tb} / \mathrm{s}$ can be achieved within the same coverage. At a ROP of $-7 \mathrm{dBm}$ and a lateral distance of $0.9 \mathrm{~m}$, the comparison of maximum capacities of all WDM channels using tracking or manual optimization is shown in Fig. 4(a). To illustrate the impact of the increased link loss imposed by sub-optimal tracking at large lateral distances, we also show the example of the bit loading on the subcarriers and the corresponding SNR profiles of the 1553.33-nm channel for the case of 0.9-m lateral distance in Fig. 4(b) and Fig. 4(c), respectively. Due to the increased link loss, fewer subcarriers can be loaded with high-order constellations, resulting in lower capacity.
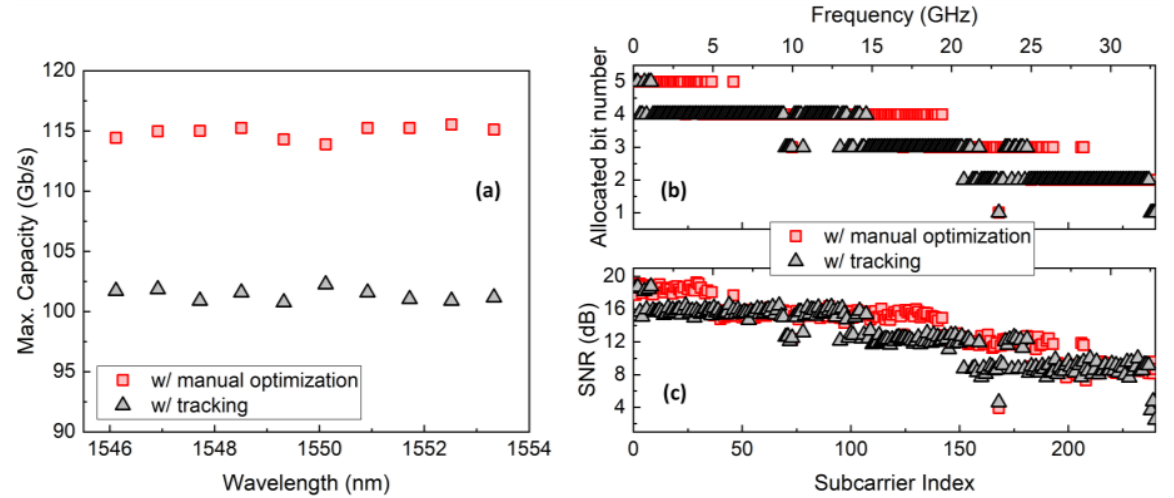

Fig. 4. (a) Comparisons between tracking and manual optimization at a ROP of $-7 \mathrm{dBm}$ and a lateral distance of $0.9 \mathrm{~m}$ : (a) maximum capacity, (b) number of loadable bits on different subcarriers and (c) SNR profiles after transmission of the 1553.33-nm channel.

\section{Conclusion}

We have demonstrated an ultrahigh 1.165-Tb/s WDM OWC transmission using a wavelength-transparent optical beam tracking and steering system. It is shown that the camera-based tracking system can achieve beyond $1-\mathrm{Tb} / \mathrm{s}$ capacity at a perpendicular distance of $3.5 \mathrm{~m}$ for a lateral coverage up to $1.8 \mathrm{~m}$. The demonstrated OWC system offers a promising solution for future ultrahigh-speed indoor wireless access networks.

This work was supported by the UK's EPSRC, through the COALESCE project (EP/P003990/1) and the European Union's Horizon 2020 research and innovation programme under grant agreement No 761329 (the WORTECS project).

\section{References}

[1] T. Koonen, et al., "High-capacity optical wireless communication using two-dimensional IR beam steering," JLT, 36(19), 4486-4493, 2018.

[2] Y. Hong, et al., "Toward user mobility for OFDM-based visible light communications," Opt. Lett., 41(16), 3763-3766, 2016.

[3] J. Wang, et al., "Terabit free-space data transmission employing orbital angular momentum multiplexing," Nat. Photonics, 6, 488-496, 2012.

[4] J. K. Doylend, et al., "Two-dimensional free-space beam steering with an optical phased array on silicon-on-insulator," Opt. Express, 19(22),

21595-21604, 2011.

[5] F. Feng, et al., "A Terabit/s indoor wide field-of-view optical wireless communication link with tracking and localization," IEEE Photon.

Technol. Lett., 2019, submitted. 